\title{
A Survey of Community Pharmacists about Work-Related Issues, How they Solve them, and who they Consult with to Solve them: Comparison of Results from the Hokkaido Area vs. a Nationwide Survey
}

\author{
Sumiyo Umeda, Hitomi Yanaguimoto, Seiichi Furuta, Nahoko Kurosawa \\ Faculty of Pharmaceutical Sciences, Hokkaido University of Science, Sapporo, Japan \\ Email: sumeda@hus.ac.jp
}

How to cite this paper: Umeda, S., Yanaguimoto, H., Furuta, S. and Kurosawa, N. (2018) A Survey of Community Pharmacists about Work-Related Issues, How they Solve them, and who they Consult with to Solve them: Comparison of Results from the Hokkaido Area vs. a Nationwide Survey. Pharmacology \& Pharmacy, 9, 447-456. https://doi.org/10.4236/pp.2018.910034

Received: September 28, 2018

Accepted: October 27, 2018

Published: October 30, 2018

Copyright $\odot 2018$ by authors and Scientific Research Publishing Inc. This work is licensed under the Creative Commons Attribution International License (CC BY 4.0).

http://creativecommons.org/licenses/by/4.0/

\section{c) (i) Open Access}

\begin{abstract}
We surveyed the drug information retrieval practices among community pharmacists in more rural Hokkaido and in entire Japan in order to explore local characteristics of work-related issues, how community pharmacists access information to address such issues, and with whom they consult to solve them. Based on the findings, we propose a strategy for improvements in similar support systems. The percentage of respondents who had experience with home care services was significantly lower in the Hokkaido group (56.0\% of 207$)$ than in the nationwide group $(70.0 \%$ of 250$)$, as was the percentage of respondents who consulted the pharmacist association and branch board (13.0\% and $20.4 \%$, respectively). The Hokkaido group also made significantly less use of websites such as d.m3.com and e.CareNet.com. The results of this survey thus indicate that the drug information retrieval in the Hokkaido group had a low implementation rate of home care services. In addition, there were low levels of utilization of the local pharmacist association, and low utilization of the websites d.m3.com and e.CareNet.com. To enhance the Hokkaido community-based integrated care system (and ones like it), we propose that it is necessary to: 1) support activities of local pharmacist associations, and 2) promote proactive implementation of drug information retrieval through education.
\end{abstract}

\section{Keywords}

Drug Informatics, Pharmacy, Drug Information Retrieval, Hokkaido, Japan 


\section{Introduction}

Community pharmacists in Hokkaido Japan provide community residents with health-care services at hospitals, nursing care facilities, or at home in collaboration with other healthcare professionals. To that end, a system to support local community pharmacists is important. As part of a continuing 10-year investigation on the information sources and retrieval practices utilized by community pharmacists based in Hokkaido, we considered several possible influences, including changes in long-term care insurance and the welfare system, an increase in home care services, preparation of generic drug information, and the spread of information technology [1].

In the present study, we conducted a survey with community pharmacists both locally in Hokkaido and nationwide in Japan regarding drug information retrieval as of 2018. It was our aim to discover the local characteristics of day-to-day drug information retrieval used by community pharmacists in Hokkaido and to utilize these findings to recommend future improvements in the support system for community pharmacists.

\section{Materials and Methods}

\subsection{Survey Target and Method: Hokkaido Area Group}

A self-administered survey of community-based pharmacists in Hokkaido was conducted between January 18 and January 31 of 2018. The questionnaire forms were mailed to 757 pharmacists working as community pharmacists in Hokkaido. Of the total, 19 questionnaires were returned as undelivered. Thus, 738 pharmacists received the questionnaire. Of the collected responses (collection rate $=32.8 \%$ ), 207 out of 242 were considered to be valid for the Hokkaido group. Respondents' consent was obtained by their indicating such on the letter of intent, and all of the responses were anonymous. This survey was assessed and approved by the research ethics committee of the Hokkaido Pharmaceutical University School of Pharmacy (approval no. 17-04-008).

\subsection{Survey Target and Method: Nationwide Area Group}

An anonymous online survey was conducted between January 31 and February 15, 2018, by the NextitResearch Institute Inc. (https://monitor.mmpr.jp/) the survey was targeted at community pharmacists or pharmacists working at drug stores nationwide who had registered with the company. The company implemented the survey by delivering a letter of intent and a questionnaire to 1000 randomly selected pharmacists who met the survey inclusion criteria. A total of 293 responses were obtained ( $29.3 \%$ of total), 283 of which were valid; the first 250 valid responses were included in the nationwide group evaluation and reported in this study. Respondents' consent was obtained by their indicating it on the letter of intent. All of the responses were anonymous. This survey was assessed and approved by the research ethics committee of the Hokkaido Pharmaceutical University School of Pharmacy (approval no. 17-04-030). 


\subsection{Survey Items}

The survey included questions regarding the type of activities that pharmacists used as a method of drug information retrieval in day-to-day work as a pharmacist, plus some basic information about the pharmacists. We were interested in identifying work-related issues, and how pharmacists solved the issues (if they did). As part of solving their work-related issues, we wondered if they consulted with anyone (and if so, who). The questionnaire contained the same questions for both the Hokkaido and nationwide groups (Figure 1).

\subsection{Analysis}

For both the Hokkaido and the nationwide groups, only fully completed questionnaires were considered valid. The Fisher's exact test was used for the comparison between the two groups in terms of the percentage of response to the questions on drug information retrieval (Q1-Q5) and the pharmacists' attributes (Q6-Q11). SPSS ver.21.0 (IBM) was used for statistical analysis. The significance of the responses was determined at $p<0.05$.

\subsection{Ethical Considerations}

For the Hokkaido group, each potential participant was sent a request to participate in the survey, which included a brief description of the study (in abstract form), some details of the personal data protection policy, and the survey questionnaire sheet. Included in the material was a section for the respondent to agree, or not, to the personal data protection policy in this study. The respondent could then choose to sign the agreement section and answer the questionnaire, or not participate. This procedure and the survey was assessed and approved by the research ethics committee of the Hokkaido Pharmaceutical University School of Pharmacy. For the nationwide group, a letter of intent was displayed on the website for the applicable target pharmacist participants, and an online survey questionnaire was conducted only for those who provided consent. As for the Hokkaido group, the survey procedure and the questionnaire was assessed and approved by the research ethics committee of the Hokkaido Pharmaceutical University School of Pharmacy. For both survey groups, it was clearly stated in the letter of consent regarding personal data that: the questionnaire should be completed anonymously, all of the responses would be encoded so that no individual could be identified, and that the survey results would be published as presentations at academic conferences and in academic papers.

\section{Results}

\subsection{Attributes of Respondents}

The results of analysis regarding the attributes of the respondents are shown in Figure 2. Of the collected responses (collection rate $=32.8 \%$ ), 207 out of 242 were considered to be valid for the Hokkaido group and 250 for the nationwide group. For the nationwide survey, 1000 community pharmacists were randomly 


\section{Questionnaire Survey Items}

Q1. What kind of problems do you have in day-to-day work as a pharmacist?

Please select a maximum of 3 applicable items.

a. Information about new or commercially available drugs

b. Information about new or commercially available quasi-drugs

c. Information about generic drugs d. Revisions to the package inserts of drugs

e. Information about the recall of quasi-drugs f. Information about the long-term care insurance and welfare system

g. Information about home care services h. Selection of references to investigate

i. Contact details for making inquiries on public holidays j. Communication with foreign patients and clients

k. Others

Q2. When you have problems in day-to-day work as a pharmacist, which method do you use to search for an answer? Please choose any applicable items.

a. Consult other pharmacists (including those at other pharmacies)

b. Consult the prefectural pharmacist association and local branch board

c. Books d. Telephone or facsimile

e. Internet f. Other method

Q3. For those who selected "d. Telephone or facsimile" in Q2, who do you contact? Please select a maximum of 3 possible contacts.

$\begin{array}{ll}\text { a. Person-in-charge of academic matters at your workplace } & \text { b. MR at a pharmaceutical company } \\ \text { c. Customer service division by the pharmaceutical company } & \text { d. Wholesaler }\end{array}$

Q4. For those who selected "e. Internet" in Q2, which websites do you use? Please select a maximum of 3 applicable items.

a. Ministry of Health, Labor and Welfare $\quad$ b. Websites of pharmaceutical companies

c. PMDA

d. m3.com

f. Risk/benefit assessment of drugs: analysis and response

g. "WAMNET" by welfare and medical service network system

h. Prefectural Health and welfare division for senior citizens

i. Self-medication database j. Foreign websites

Q5. For those who selected "e. Internet" in Q2, which method do you use to connect to the Internet? Please choose maximum 3 applicable items.
a. Computer at work
b. Personal computer
c. Smartphone
d. Tablet e. Others

Thank you for completing the questionnaire. For statistical analysis purpose, please provide the following information about yourself.

Q6. Sex

a. Male

b. Female

Q7. Age range

$\begin{array}{llllll}\text { a. } 20 \mathrm{~s} & \text { b. } 30 \mathrm{~s} & \text { c. } 40 \mathrm{~s} & \text { d. } 50 \mathrm{~s} & \text { e. } 60 \mathrm{~s} & \text { f. } 70 \mathrm{~s}\end{array}$

Q8. Years of experience as a pharmacis

$\begin{array}{lllll}\text { a. } 1-5 . & \text { b. } 6-10 & \text { c. } 11-15 & \text { d. } 16-20 & \text { e. }>20\end{array}$

Q9. Management type at your workplace

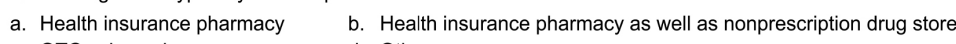

c. OTC sales only d. Others

Q10. Business type at your workplace

$\begin{array}{lll}\text { a. Franchised } & \text { b. Individual business } & \text { c. Others }\end{array}$

Q11. Experience with home care services

$\begin{array}{ll}\text { a. Yes } & \text { b. No }\end{array}$

Figure 1. The survey of community pharmacists about work-related issues, how they solve them, and who they consult with to solve them.

selected from among the total registrants. A total of 293 responses were obtained (29.3\%), 283 of which were valid; the first 250 valid responses were included in the nationwide group in this study.

\subsubsection{Sex and Age}

Male respondents accounted for 93 (44.9\%), female respondents 114 (55.1\%) of the Hokkaido group, and 148 (59.2\%) and 102 (40.8\%), respectively, for the nationwide group (significant difference between groups, $p<0.005$; Figure 2(a). In 

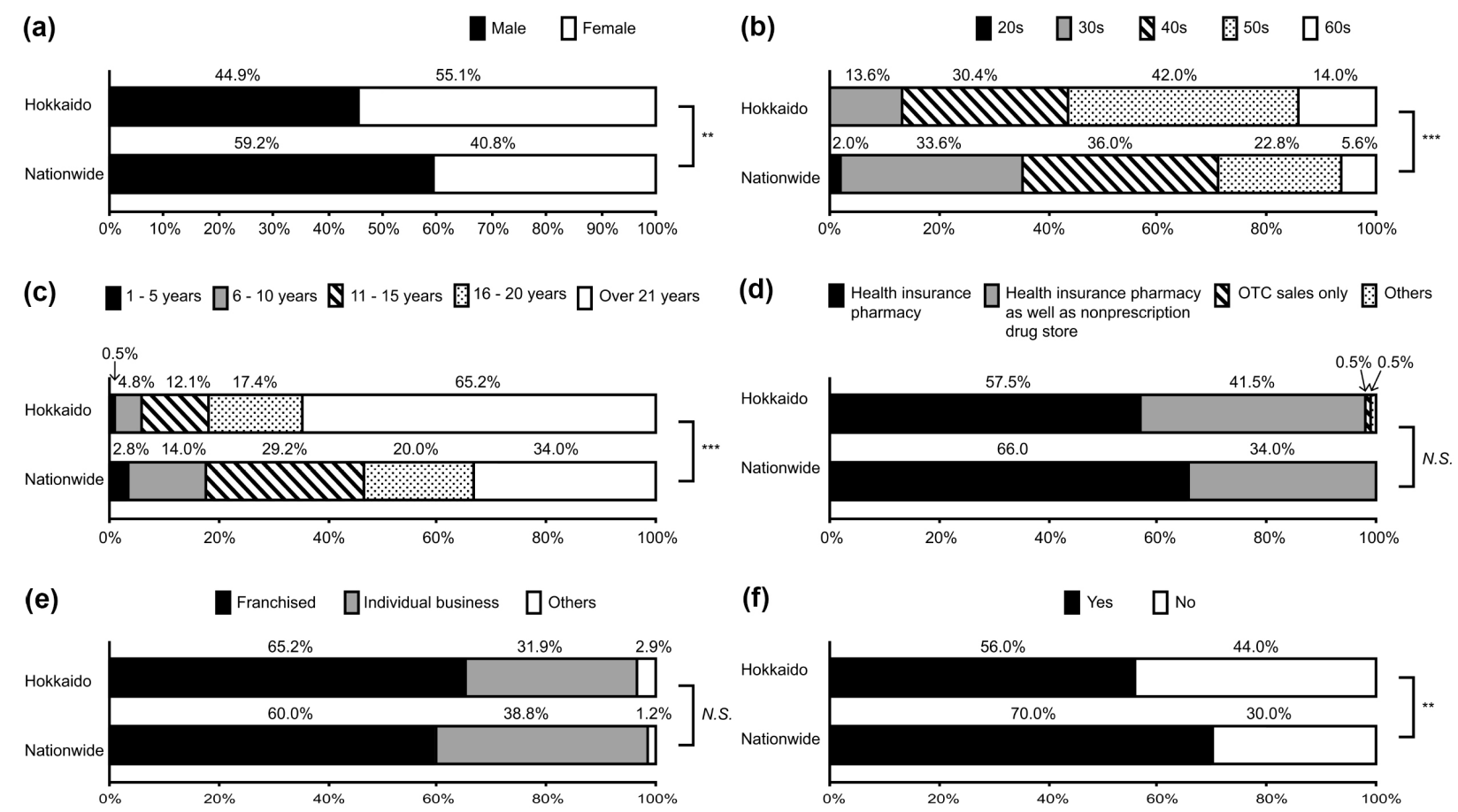

Figure 2. Attributes of respondents. (a) Sex (Q6), (b) Age (Q7), (c) Years of experience as a pharmacist (Q8), (d) Management type (Q9), (e) Business type (Q10), and (F) Experience of home care services (Q11). Hokkaido group $(\mathrm{n}=207)$ vs nationwide group $(\mathrm{n}=250) .{ }^{* *} p<0.005,{ }^{* * *} p<0.001$ : Fisher's exact test.

the Hokkaido group, the largest \% respondents were in their 50s $(42.0 \%)$, then those in their 40 s $(30.4 \%)$. In the nationwide group, the largest $\%$ respondents were in their $40 \mathrm{~s}(36.0 \%)$, then those in their $30 \mathrm{~s}(33.6 \%)$. The groups were significantly different $(p<0.001$; Figure $2(b))$.

\subsubsection{Years of Experience as a Pharmacist}

In the Hokkaido group, $65.2 \%$ of the respondents had more than 21 years of experience, followed by $17.4 \%$ with 16 - 20 years of experience. In the nationwide group, $34.0 \%$ had more than 21 years of experience; $29.2 \%$ had $11-15$ years of experience. The two groups were significantly different $(p<0.001$; Figure $2(c))$.

\subsubsection{Management Type}

In the Hokkaido group, $57.5 \%$ of the respondents' work places function solely as health insurance pharmacies; and $41.5 \%$ function as both a health insurance pharmacy and as a nonprescription drug store. In the nationwide group, these percentages were $66.0 \%$ and $34.0 \%$, respectively. There was no significant difference between the two groups (Figure 2(d)).

\subsubsection{Business Type}

In the Hokkaido group, $65.2 \%$ of respondents worked at franchised stores and $31.9 \%$ ran their own businesses; in the nationwide group the proportions were $60.0 \%$ and $38.8 \%$, respectively. There was no significant difference between the two groups (Figure 2(e)). 


\subsubsection{Experience with Home Care Services}

The percentage of respondents who answered "Yes" to the question on whether they had experience of working with home care services was significantly $(p<$ $0.005)$ smaller in the Hokkaido group (56.0\%) than in the nationwide group (70.0\%; Figure 2(f)).

\subsection{Questions}

\subsubsection{Issues in Day-to-Day Work as a Pharmacist (Q1)}

Item " $i$ ", regarding requests to provide contact details for enquiries made on public holidays, was selected by $10.1 \%$ of the respondents in the Hokkaido group and $20.0 \%$ of the respondents in the nationwide group. There was a significant difference between the two groups $(p<0.005)$. The responses for all other choices in Q1 showed no significant differences (Figure 3(a)).

\subsubsection{Investigation Methods about Q1 (Q2)}

Item " $\mathrm{b}$ ", regarding consulting the pharmacist association and the local branch board, was selected significantly $(p<0.05)$ less frequently by the Hokkaido group (13.0\%) than by the nationwide group (20.4\%). Item "d", regarding use of telephone or facsimile, was selected by the Hokkaido group approximately twice as often (52.2\%) compared with the nationwide group (25.6\%; $p<0.001$; Figure $3(\mathrm{~b}))$.

\subsubsection{Telephone or Facsimile Numbers of whom to Contact (Q3)}

There were no significant differences between Hokkaido and nationwide groups (Figure 4(a)).

\subsubsection{Frequently Used Websites (Q4)}

The Hokkaido group reported a significantly less use of websites such as d.m3.com than did the nationwide group ( $13.5 \%$ versus $34.3 \%$; $p<0.005)$. Similarly, the use of the website e.CareNet.com by the Hokkaido group was approximately one-third the use by the nationwide group (3.6\% versus $10.9 \%$; $p<$ 0.001 ; Figure 4(b)).

\subsubsection{Methods to Connect to the Internet (Q5)}

There were no significant differences between Hokkaido and nationwide groups (Figure 4(c)).

\section{Discussion}

With regard to the characteristics of the survey respondents, the male:female ratio in the Hokkaido group was approximately 1:1.2, which closely reflects the sex ratio of pharmacy graduates of our University [2]. The sex ratio in the nationwide survey group was $1.4: 1$, which is different from the ratio of community pharmacists nationwide, where a larger proportion of pharmacists nationwide are female (approximately 1:2) [3]. This difference could be the result of sex difference with respect to accessing the online questionnaire; however, this possibility remains unproven. With regard to age, the Hokkaido group had a larger 
(a)
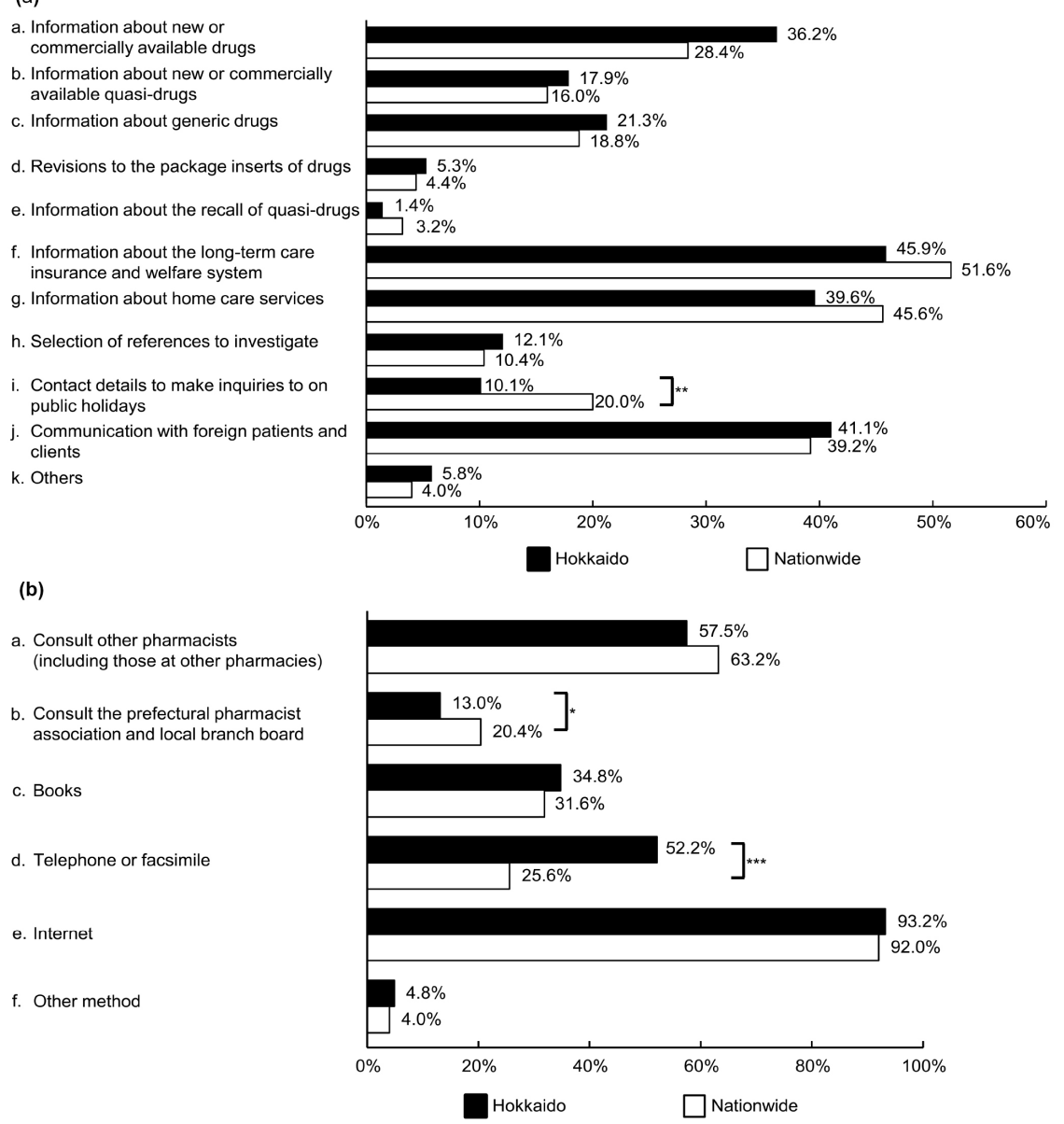

Figure 3. Questions about daily work as a pharmacist. (a) Issues in day-to-day work as a pharmacist (Q1). (b) Investigation methods for the issues in day-to-day work as a pharmacist mentioned in Q1 (Q2). Hokkaido group $(\mathrm{n}=207)$ vs nationwide group $(\mathrm{n}=250)$. ${ }^{\star} p<0.05,{ }^{* *} p<0.005,{ }^{* *} p<0.001$ : Fisher's exact test.

percentage of respondents in their 40s or older than did the nationwide group. This might be attributable to the introduction of the 6-year system of pharmaceutical education, and decreasing sense of belonging among recent graduates. Alternatively, conducting the questionnaire online for the nationwide group might have produced a bias favoring younger respondents. Although the Hokkaido group reported a larger percentage of respondents with more experience than the nationwide group, this could simply reflect the age range of the respondents. For management type and business type, the absence of significant differences in both answers indicates that the pharmacy structure in Hokkaido is similar to the national average. With regard to experience with home care services, the number of respondents in the Hokkaido group who answered "Yes" to the question was significantly lower than that in the nationwide group. The total number of home medical treatment support hospitals and clinics per 100,000 individuals aged greater than 65 years is 47.2 nationwide and 24.4 in Hokkaido [4]. Thus, our results corroborated the current situation. 
(a)

a. Person-in-charge of academic matters
at your workplace
b. MR at a pharmaceutical company
c. Customer service division at
pharmaceutical company
d. Wholesaler
(b)
a. Ministry of Health, Labor and Welfare
b. Websites of pharmaceutical companies
c. PMDA
d. m3.com
e. Care Net.com
f. Risk/benefit assessment of drugs,
analysis and response
g. "WAMNET" by welfare and medical
service network system
h. Health and welfare division of the
jorion database
(bebsites

(c)

a. Computer
b. Tablet
c. Smartphone
d. Others
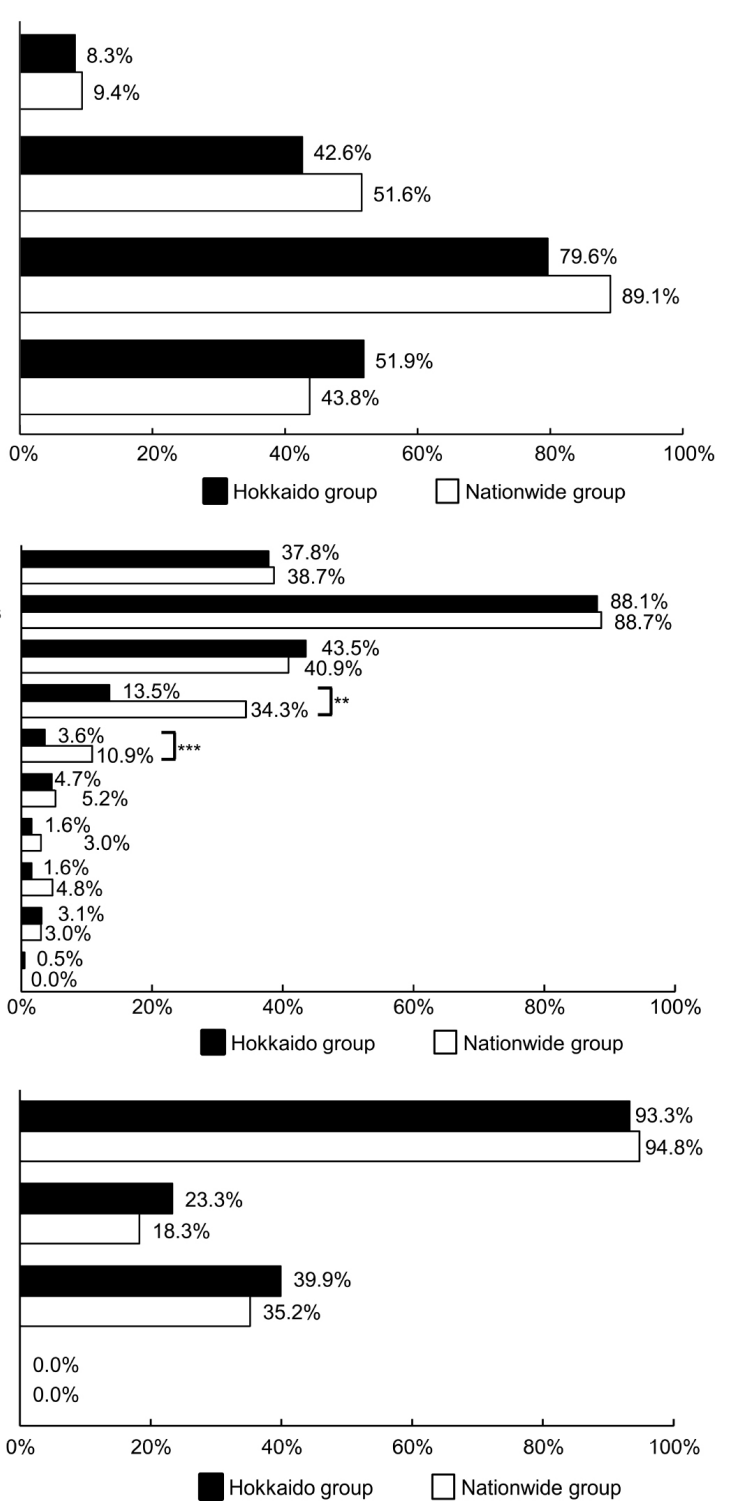

Figure 4. Questions regarding Q2. (a) Who should be contacted to solve issues in day-to-day work as a pharmacist for those answering "telephone or facsimile" in Q2 (Q3). Hokkaido group $(\mathrm{n}=108)$ vs nationwide group $(\mathrm{n}=64)$. (b) Which websites are used to solve issues in day-to-day work as a pharmacist for those answering "Internet" in Q2 (Q4). Hokkaido group $(\mathrm{n}=193)$ vs nationwide group $(\mathrm{n}=230)$. (c) Which method is used to connect to the Internet to solve issues in day-to-day work as a pharmacist for those answering "Internet" in Q2 (Q5). Hokkaido group $(\mathrm{n}=193)$ vs nationwide group $(\mathrm{n}=230) .{ }^{* *} p<0.005,{ }^{* *} p<0.001$ : Fisher's exact test.

The Hokkaido group had a significantly smaller number of responses to Q1 item " $i$ " regarding whether they had problems about whom to contact with inquiries on public holidays. This was in accordance with a survey conducted in February 2017 that stated that $77.4 \%$ of all health insurance pharmacies in Hokkaido have systems to accommodate client queries on a 24-h basis [5]. The result also reflected that there are more pharmacies nationwide in which only one pharmacist works full-time) [6]. 
Regarding the investigation methods about Q1 (Q2), significantly fewer respondents in the Hokkaido group selected " $b$ " (consult the prefectural pharmacist association and local branch board). The activities and benefits of local associations, such as cooperation with professionals in other disciplines and promotion of team medicine, play a crucial role in the local comprehensive care system. As one example, the Hokkaido pharmacist association provides continuous education for pharmacists and publishes information on community healthcare and medical services provided at home [7]. Universities should thus support the pharmacist association and local branch board in Hokkaido and guide their students and graduates to utilize information regarding cooperation in the community. In terms of the use of Internet and devices used for accessing the Internet, there was no significant difference between the Hokkaido and nationwide groups. However, the Hokkaido group used telephone and facsimile approximately twice as often as the nationwide group, which may be related to the age difference of respondents.

In terms of frequently used websites, the Hokkaido group reported significantly lower usage of medical content services with membership systems, for example d.m3.com and e.CareNet.com, than did the nationwide group. Although the reasons for this remain unclear, these websites contain a wide range of useful medical information, and we believe that it is important in the future to enhance guidance at universities on their optimum utilization. Specific examples of the university's efforts to stimulate drug information retrieval by community pharmacists in Hokkaido include: 1) recommending the use of drug information websites and introducing how to use them, and 2) delivering lectures on how community healthcare should be managed in collaboration with the pharmacist association. Logical development from the present findings is limited because of paucity of previous reports dealing specifically with community pharmacists in Hokkaido. In the future, issues that community pharmacists in Hokkaido face may be identified by enhancing the questions to ask specific factors based on the present findings.

The major limitation of this study was that the questionnaires were administered in a different manner: the questionnaire was sent to community pharmacists in Hokkaido by mail, whereas the nationwide survey of community pharmacists was administered online. Significant differences depending on age and number of years experience as a community pharmacist were observed, which may have resulted from how the questionnaires were administered.

\section{Conclusions}

This survey revealed the local characteristics of drug information retrieval in Hokkaido, which is a more sparsely populated region of Japan, such as a lower implementation of home care services compared with a nationwide group, lower utilization of local pharmacist associations, higher utilization of telephone and facsimile, and lower utilization of internet websites such as d.m3.com and e.CareNet.com. Educational services provided by universities include publishing 
an online listing of websites useful for problems encountered by community pharmacists and describing how to use them. Another method is to host training seminars several times a year wherein participants receive practical training on how to use computers for the purpose of collecting information regarding problems related to community pharmacists' work. It is also clear that in Hokkaido the use of home care services is behind as compared with the whole country. In a time of an aging society, it is necessary for the community pharmacists in the region to be engaged in home care services positively.

The results of the present study demonstrate that to enhance local comprehensive care systems in the future, it is necessary to support the activities of local pharmacist associations and promote more proactive implementation of drug information retrieval through education.

\section{Conflicts of Interest}

The authors declare no conflicts of interest regarding the publication of this paper.

\section{References}

[1] Umeda, S., Yanaguimoto, H., Furuta, S. and Kurosawa, N. (2018) Survey of Drug Information Retrieval Situation by Community Pharmacist in Hokkaido Area-Transition in the Last Decade. Japanese Journal of Community Pharmacy, 6 , 23-35.

[2] Hokkaido College of Pharmacy (2004) 26. Statistical Reference (Educational \& Administration Affairs). Keisei Commemorative Issue for $30^{\text {th }}$ Anniversary of Hokkaido College of Pharmacy 316.sd.

[3] Ministry of Health, Labor and Welfare (2017) General Situation of Survey in 2016 for Doctors, Dentists and Pharmacists. https://www.mhlw.go.jp/toukei/saikin/hw/ishi/16/dl/gaikyo_b1.pdf

[4] Ministry of Health, Labor, and Welfare (2017) By-Region Data Collection Regarding Home Healthcare (Renewal Date: Nov. 22, 2017). https://www.mhlw.go.jp/file/06-Seisakujouhou-10800000-Iseikyoku/0000185771.xls

[5] Yakuji Nippo. (2018) 60\% of Pharmacies Claiming for Guidance Fee for Regular Pharmacist-Survey for Pharmacies in Hokkaido Reveals 20\% of Pharmacies Not Handling Over-the-Counter Drugs. https://www.yakuji.co.jp/entry58226.html

[6] Cabinet Office. (2017) Analysis regarding Cost Structure and Tendency of Drug Dispensation and Drug Cost. Policy Issue Analysis Series No. 14, 42-43. http://www5.cao.go.jp/keizai3/2017/08seisakukadai14-0

[7] Hokkaido Pharmacist Association (2018) Introduction. http://www.doyaku.or.jp/index.html 